\title{
Psychological Effects of MDE in Normal Subjects
}

\section{Are Entactogens a New Class of Psychoactive Agents?}

Leo Hermle, M.D., Manfred Spitzer, M.D., Ph.D., Dieter Borchardt, Karl-Artur Kovar, Ph.D., and Euphrosyne Gouzoulis, M.D.

The so-called entactogens 3,4-methylenedioxymethamphetamine ( $(M D M A]$ also known as "Ecstasy," or "Adam") and its analog 3,4-methylenedioxyethamphetamine ([MDE] also known as "Eve") exert similar psychotropic effects in humans. Two double-blind placebo-controlled psychometric studies with normal control subjects were conducted. Placebo or MDE (140 mg) was administered orally to eight male volunteers at 1:30 P.M. and to six subjects ( 3 male, 3 female) at 11 P.M. Psychologic tests and clinical ratings were performed 1 hour before the administration of the drugs, as well as 2, 5, and 24 hours after drug intake and 7 days thereafter in the first study. In the second study, measures were taken at times $-1,+8.5,+24$ hours, and +7 days. The majority of the psychotropic effects resembled those that have already been described in anecdotal reports. The substance produced a partially controllable state of enhanced insight, empathy, and peaceful feelings. All subjects displayed a general stimulation with increased psychomotor drive, logorrhea, and facilitation of communication. One of the fourteen volunteers developed a toxic psychosis. One volunteer displayed a dysphoric reaction, one suffered from episodes of anxiety for some days after the experiment. The findings support the hypothesis that MDMA and MDE represent a novel pharmacologic class. [Neuropsychopharmacology 8:171-176, 1993]
KEY WORDS: Entactogens; MDE; MDMA; Psychologic effects

According to anecdotal evidence, 3,4-methylenedioxymethamphetamine ([MDMA] also known as "Ecstasy," "Adam," and "XTC") and 3,4-methylenedioxyethamphetamine ([MDE] also known as "Eve") exert unique psychologic effect in humans, discriminating them from chemically related substances like the stimulant amphetamine and the hallucinogens 3,4-methylenedioxyamphetamine (MDA) and 3,4,5-trimethoxy-phene-

From the Department of Psychiatry, Christophsbad, Göppingen (LH), Department of Psychiatry, University of Heidelberg (MS), Institute of Pharmaceutics, University of Tübingen (K-AK), and Department of Psychiatry, University of Freiburg (DB, EG), Germany.

Address reprint requests to: Leo HermIe, M.D., Department of Psychiatry, Christophsbad, Faurndauerstrasse 16, D-7320 Göppingen, Germany.

Received January 28, 1992; revised April 14, 1992 and June 10, 1992; accepted June 18, 1992. thylamine ([mescaline] Shulgin 1986; Nichols 1986). Although MDMA was assigned to a Schedule I status by the Drug Enforcement Agency in 1985, there is an ongoing discussion about its potential uses in psychotherapy and the possible adverse neurotoxic effects it may have (Beck 1990; Peroutka 1990; Battaglia et al. 1988; Ricaurte et al. 1988; Gibb et al. 1990; Schmidt et al. 1987). After the scheduling of MDMA, its N-ethyl analog, MDE, served as an substitute for recreational users (Beck 1990).

Like MDMA and MDA the N-ethylated MDE has a high affinity at reuptake sites of central catecholaminergic, dopaminergic, and serotonergic neurons and is taken up via a fluoxetine-sensitive carrier mechanism. These substances cause reuptake inhibition and release of the mentioned transmitters (Johnson et al. 1988).

Neurotoxicity studies of MDMA and MDE found a depletion of serotonin and long-term axon damage after the acute or chronic administration of these agents 
in a wide dose range (Stone et al. 1987; Battaglia et al. 1988; Ricaurte et al. 1987). Studies found MDE to be less neurotoxic than MDMA (Schmidt et al. 1987).

Various nonsystematic observations suggest that entactogens are different from hallucinogenic drugs. There are no reports of individuals or drug-addicts who take frequent or large amounts of MDMA or MDE for extended periods of time. The reason for this is that the "positive" effects of the drug seem to diminish and the "negative" effects seem to increase with time (Greer et al. 1986). Data on the psychologic effects of MDMA and MDE are limited. Although there are some reports of panic reactions and "bizarre and risky behavior" (Dowling et al. 1986) during peak intoxication from the drug, MDMA and MDE enjoy a reputation for producing a well-controllable emotionally peaceful experience with enhanced insight, empathy, and closeness to others with only rare occasions of severe toxicity. More chronic psychologic problems such as delayed anxiety disorders have been observed in a few individuals following an MDMA experience (Seymour 1986). These findings underscore a potential danger of recreational use or "selftherapy" by individuals who run the risk of exacerbating emotional problems.

It was hypothesized, that MDMA and MDE might constitute a novel pharmacologic class called "entactogens." Nichols (1986) gives the following defınition of this new term, " . . . it seemed that the Latin root of this word, "tactus," would be appropriate as part of the term. Addition of the Greek roots en (within or inside) and gen (to produce) created the term entactogen, having the connotation of producing a touching within." From a therapeutic viewpoint, it should be noted that antidepressant and anxiolytic properties have been reported (Greer et al. 1986).

To our knowledge, this is the first report of a controlled investigation of the psychologic effects of MDE. Data from two separate studies, one study on neuroendocrine and cardiovascular effects, and a second study on the effects of MDE on sleep electroencephalography (EEG) were evaluated. The EEG study was done in order to specify the effects of MDE on sleep patterns that are known to be closely connected with certain neurotransmitters. The leading idea of the study was that a distinct sleep pattern distinguishing MDE from amphetamine and hallucinogens might be detectable. The neuroendocrine, cardiovascular, and sleep-EEG data are reported elsewhere (Gouzoulis et al. 1992a,b, in press).

\section{MATERIALS AND METHODS}

\section{Subjects and Study Design}

Study 1. In a randomized double-blind placebo-controlled cross-over study, eight physically and mentally healthy male volunteers ranging in age from 29 to 50 years (mean 38 years) were included. Subjects were selected 3 to 7 days before the experiment by screening with the structured clinical interview for DSM-III-R (German version, Wittchen et al. 1987) to ensure that they had no personal or family history of psychiatric disorders in first degree relatives. In addition, the Freiburger Personality Inventory (FPI), the Vegetative Lability Scale (BL), and the State-Trait Anxiety Scale (STAI)- $\mathrm{X}_{2}$ were performed at this time. To exclude physical disorders, subjects were screened by means of physical examination, EEG, and electrocardiography. All subjects were academics (seven physicians and one engineer), were fully informed of the toxicology study results at the time of their participation, and gave written informed consent.

Study 2. In a similar design, six healthy volunteers ( 3 male, 3 female) ranging in age from 28 to 33 years (mean 29.5 years) participated. All participants were academics (five physicians and one psychologist). In the female subjects, pregnancy was ruled out by an appropriate test.

All 14 subjects had been without any medication for at least 3 months prior to the study, and had not been subject to excessive alcohol or caffeine intake, stressful life events, shift work, or time shifts greater than 3 hours during the preceding 3 months. None of the subjects met the DSM-III-R criteria for alcohol or substance abuse. None of the subjects had previous experiences with MDE. Two subjects each reported one single experience with MDMA, two subjects had one or two previous experiences with hallucinogens some years ago. The studies were approved by the local ethics committee at the University of Freiburg, Germany.

\section{Substances}

The MDE used in this study was synthesized at the Institute of Pharmaceutics, University of Tübingen, and was obtained in the form of $70 \mathrm{mg}$ capsules. Placebo capsules were obtained from the same institute. Each subject received $140 \mathrm{mg}$ MDE ( 2 to $3 \mathrm{mg} / \mathrm{kg}$ of body weight), a dose commonly taken for recreational and therapeutical use (Seymour 1986; Beck 1990). It should be noted that quantitative data on the pharmacokinetic aspects of MDE are not yet available. In particular, nothing is known about its bioavailability after oral ingestion under fasted and nonfasted conditions in humans. From the pattern of metabolites that have been detected in the urine, the existence of two metabolic pathways (O-desmethylization and $\mathrm{N}$-desalkylization) in human beings can be inferred. All metabolites are excreted as sulphate and/or glucuronide in the urine (K-A Kovar, unpublished data). 


\section{Psychometric Measurements}

All subjects were rated by two experienced psychiatrists with the Manic-State Rating Scale ([MSRS] Beigel et al. 1971). To evaluate the general psychologic effects of the drug, the following self-rating scales were used: the FPI revised (Fahrenberg et al. 1984); the STAI-X ${ }_{1}$ (Laux et al. 1981); the Depression Scale ([DS] von Zerssen 1986); the Vegetative Lability Scale (BL) (von Zerssen 1986); the Adjective Mood Scale, "Befindlichkeitsskala" ([BFS] von Zerssen 1986); a questionnaire for the assessment of "Altered States of Consciousness" (APZ), with its dimensions oceanic boundlessness (OSE); dread for ego-dissolution (AIA); and visionary restructuralization (VUS) (Dittrich 1985).

For the evaluation of cognitive functions, the following tests and measures were used: the Benton Test (Instruction A, Benton 1971); repetition of numbers forward and backwards (Hamburg-Wechsler Intelligence Evaluation [HAWIE] subtest 3, 1964); and counting backwards ( 100 minus 7 , in a series, until reaching 51 ). In addition to these measures, two semistructured interviews (self-reports) were obtained 1 day and 1 week after the experimental session.

\section{Experimental Procedure}

Study 1. Subjects arrived at the hospital at 12:00 A.M. and received a standardized calorie- and electrolytebalanced diet for lunch. Beginning at 1:00 P.M., subjects had to rest supine on a bed in a single room while they were observed by a video camera with a monitor located in the adjacent observation unit. They were afforded the opportunity to communicate with investigators by intercom. Either MDE or placebo was given orally with some liquid at 1:30 P.M. Psychometric tests were performed before the ingestion of MDE or placebo, as well as 2, 5, and 24 hours after ingestion of the drug, and again 7 days later.

Study 2. Subjects spent a total of 4 nights in the sleep laboratory, 1 adaptation night and 1 consecutive night while taking MDE (140 mg orally) or placebo. Two to 6 weeks later, subjects spent another adaptation night and another consecutive night taking either placebo or MDE (crossover). During the 2 weeks preceding the sleep EEG examination, each subject was instructed to go to bed between 10:30 P.M. and 11:30 P.M. This was done in order for the subjects to become acclimated to the "lights off" time. Drugs were administered at 11:00 P.M., just before lights were switched off. Observation of the subject was possible by infrared video camera, and communication was made possible by intercom. Psychometric tests were performed before drug intake (at 10:00 P.M.), after awakening (at 7:30 A.M.), as well as 24 hours and 7 days after drug intake.

\section{RESULTS}

\section{Study 1}

Trait measures: under baseline conditions, results of the FPI-R were in the normal range, except for the dimension of "openness," in which a higher than average mean score was obtained. The STAI- $\mathrm{X}_{2}$ yielded normal results. State measures: in the MSRS, a significant difference between MDE and placebo was found at time 1 (2 hours after MDE intake, $p=0.005$, Student's $t$-test two-tailed, see fig. 1).

In the STAI- $X_{1}$, no significant difference between MDE and placebo was found. The analysis of individual state anxiety scores, however, showed a decrease of anxiety in seven of the eight subjects. One subject (number 8), however, displayed a marked increase of anxiety under the influence of MDE. In the semi-structured interview, this subject described this state as follows, "The onset of the effect is rapid. Within seconds, clouds appeared within my head, and afterwards there was a marked lack of concentration. It was impossible to read. Bits and pieces of thought pass by but cannot be put together. This state of restlessness and lack of coordination was experienced as markedly unpleasant and senseless. The accompanying somatic phenomena were also experienced as unpleasant: restlessness, palpitations, hyperventilation, tickling paresthesia in the fingers, dry mouth, tension in the muscles around the mouth." As can be seen from this self-report, somatic symptoms accompanying generalized anxiety were markedly present. No significant correlation between state and trait anxiety, as measured by STAI- $\mathrm{X}_{2}$ and STAI- $X_{1}$ was found. No significant difference between $\mathrm{MDE}$ and placebo was found on the DS, BFS, or BL

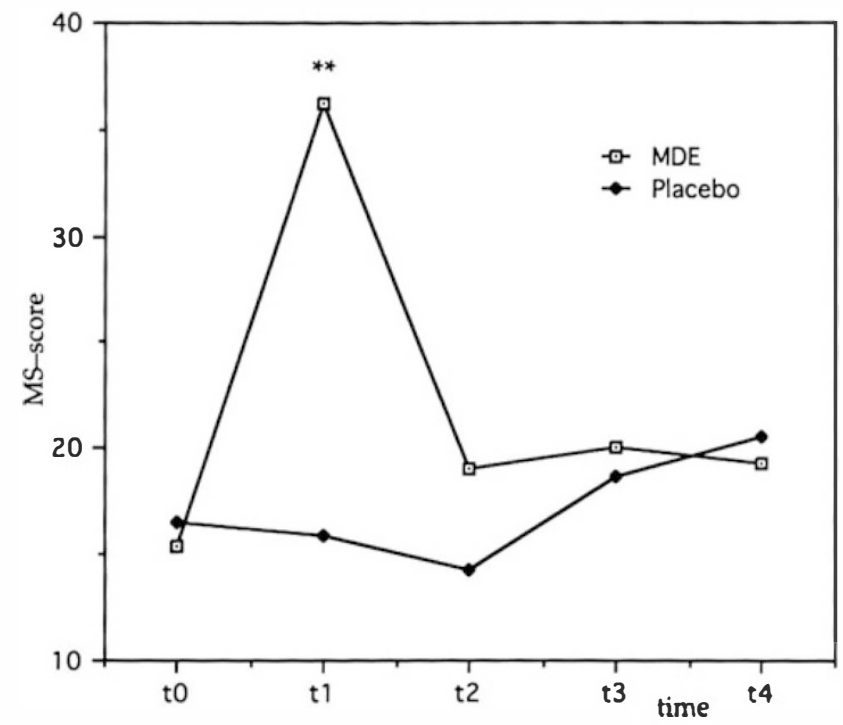

Figure 1. Ratings using the MSRS while subjects were under the influence of MDE or placebo. Asterisks indicate: $p=$ 0.005 . 
scales. No significant difference in the measures of cognitive functioning (Benton, HAWIE subtest of counting backwards, or subtraction task) was found between MDE and placebo groups.

\section{Study 2}

All subjects fell asleep about 30 minutes after "lights off," awoke after they had slept for 30 to 60 minutes, and stayed awake for at least 150 minutes. Three of the subjects reported experiences of depersonalization, such as a "light feeling in the body," a "sense of not laying in bed but rather floating in the air," a feeling "as if the clock has come to a standstill," and an experience "as if the eyes were sticking out of the head." Two subjects noted "mystic" experiences. One subject developed a psychotic state about 1 hour after the intake of MDE, which lasted for about $2 \frac{1}{2} 2$ hours. In this state, he suffered from vivid auditory and visual hallucinations, delusions of reference, and loss of control of thought. His psychotic symptoms were completely abated without the use of any medication when assisted by a clinician who "talked him down" (for a detailed report, see Gouzoulis et al. 1992c). One subject suffered from depressive affect of 30 minutes duration in the 5 days following the experiment. No difference between $\mathrm{MDE}$ and placebo was found in any of the ratings (BL, BFS, STAI- $X_{1}$, or DS).

\section{Studies 1 and 2}

Data from the APZ questionnaire of the two studies were pooled $(n=14)$. As can be seen from Table $1, \mathrm{MDE}$ produced highly significant differences compared to placebo in all three scales.

The semi-structured interviews revealed that the drug intake influenced the subjects' attitudes for at least 7 days. Some subjects felt "enlightened" by the experience, and reported an increased interest in questions regarding their attitudes towards life, self, general aims, and interpersonal relationships.

\section{DISCUSSION}

Standard psychometric methodology and a doubleblind placebo-controlled cross-over study design was used to obtain data on the psychologic effects of MDE, an agent that has been suggested to represent a new class of psychoactive substances labeled entactogens, in 14 normal subjects. The major effects of MDE were a reduction of anxiety, an increased drive with pronounced partly euphoric and partly depressed mood, and an improved responsiveness in, and openness to, communication. Psychotic symptoms were present in only one subject, who suffered from a schizophrenia-
Table 1. Breakdown of APZ Scores for Subjects Under the Influence of MDE and Placebo

\begin{tabular}{lccc}
\hline & $\begin{array}{c}\text { MDE } \\
(\boldsymbol{n}=\mathbf{1 4})\end{array}$ & $\begin{array}{c}\text { Placebo } \\
(\boldsymbol{n}=\mathbf{1 4})\end{array}$ & $(\boldsymbol{p})$ \\
\hline OSE & $3.92 \pm 2.97$ & $0.14 \pm 0.36$ & 0.000 \\
AIA & $2.57 \pm 3.08$ & $0.07 \pm 0.26$ & 0.008 \\
VUS & $1.64 \pm 1.86$ & $0.00 \pm 0.00$ & 0.006 \\
\hline
\end{tabular}

Data are reported \pm SD.

like psychotic episode for $2^{1 / 2}$ hours. Three subjects experienced episodes of depersonalization and derealization, and two subjects reported "mystical" experiences. For several days, one of these th: subjects developed a depressive state, including episodes of anxiety.

Although most anecdotal reports state that MDMA and MDE are "safe" drugs, dysphoric reactions, panic attacks (Whitaker et al. 1989; Seymour 1986), and "bizarre and risky behavior" (Dowling et al. 1987) were reported in single cases of entactogen-users. However, these reports are difficult to interpret, as the effects might have been caused by interactions with other ingested substances, such as alcohol. Furthermore, the precise chemical qualities of the substances used were unknown because of their illegal production. Our data suggest that these anecdotal reports represent valid observations of the effects of the substance, i.e., that entactogens may exert psychotomimetic effects. Moreover, the frequency of such serious toxic reactions may have been underestimated by those authors who made estimates by merely relying upon assumptions about the frequency of use and the frequency of respective reports.

It is noteworthy that the more severe psychologic reactions occurred in the second experiment in which the subjects took the substance at bedtime and woke up spontaneously (from deep sleep) about one hour later. As it is generally known that the effects of psychoactive drugs are highly dependent on the setting in which they are taken, it is likely that the severity of the substance-induced changes can be attributed to the special setting adopted in the second study.

As it is generally known that the effects of psychoactive substances are dependent upon the set, i.e., personal factors (including educational level), and as all subjects were professional people from a medical environment, we are not able to comment on the generality of our findings with respect to subjects from different socioeconomic and educational backgrounds.

The hitherto anecdotally reported powerful antidepressant and anxiolytic properties of MDE seem to be different from the effects of standard antidepressant and anxiolytic agents. According to our data, MDE produced a decrease in anxiety and a increase of euphoric 
and depressed mood with an augmented drive being present at the same time. This seemingly paradoxical finding of both emotional states, manic-like temper and depressed mood, may be regarded as the result of a general increase in responsiveness to emotions. Under the influence of MDE, these states were experienced in a calm and relaxed manner, almost without any accompanying anxiety. Moreover, the fact that MDE does not lead to a marked reduction of general sensitivity and cognitive functioning, may add to the emotional responsiveness of the subjects.

Unlike hallucinogenic drugs, MDE did not disturb perception, formal thought processes, or memory, nor did it produce psychotic symptoms such as hallucinations, with the exception of one case. Although all the scales of the APZ questionnaire revealed significant drug effects, these effects were mainly concerned with the dissolution of ego-boundaries, experiences of derealization, and feelings of calmness and happiness, often mixed with anxious passivity experiences. Furthermore, drug sessions influenced some of the subjects' perspective on matters of everyday life for at least a week after drug intake in a way that may be considered "therapeutic" in some cases. The summary results of this psychometric study, in part, confirmed what was known about the effects of MDE from anecdotal reports. However, a more precise picture emerges from our data. The entactogens induce pronounced mental effects which do not closely resemble those of hallucinogenic agents. Hence, it may be concluded that they represent a new class of psychoactive substances. Despite reports that entactogens are generally safe, and even despite the conceivable beneficial effects, our data suggest that the safety of these agents may depend upon the setting in which the substances are used.

Generally speaking, the study of psychoactive substances under controlled laboratory conditions represents a research strategy that merits further inquiry for several reasons. As a methodologic tool, experimental psychosis can be studied with a broad array of psychologic as well as biologic techniques. From a systematic perspective, the study of the effects of psychoactive substances enhances our general understanding of psychiatric phenomena, from subtle subjective psychopathological experiences to the underlying biologic brain mechanisms, and even allows correlational analyses, bridging the "gap" between the mental and the physical. Lastly, from the point of view of public health, it is of utmost importance to society to have access to valid knowledge about the effects of substances that are widely used by the general public.

\section{REFERENCES}

Battaglia G, Brooks BP, Kulsakdinum C, De Souza EB (1988a): Pharmacologic profile of MDMA (3,4-methylenedioxy- methamphetamine) at various brain recognition sites. Eur J Pharmacol 149:159-163

Battaglia G, Yeh SY, De Souza EB (1988b): MDMA-induced neurotoxicity: Parameters of degeneration and recovery of brain serotonin neurons. Pharmacol Biochem Behav 29:269-274

Beck J (1990): The public health implications of MDMA use. In Peroutka SJ (ed), Ecstasy: The Clinical, Pharmacological and Neurotoxicological Effects of the Drug MDMA. Boston, Kluwer, pp 77-103

Beigel A, Murphy DL, Bunney WE (1971): The Manic-State Rating Scale. Arch Gen Psychiatry 25:256-262

Benton AL (1972): Der Benton-Test. Handbuch. Stuttgart, Huber

Dittrich A, von Arx S, Staub S (1985): In collaboration with: Cochrane R, Cordero M, Davenport D, Davenport RH, Deters H, Dierse B, O'Callaghan MAJ, Pollonio P, Pusterla-LongoniC, Ratti A, Simmen R, and Simoes M. International Study on Altered States of Consciousness (ISASC). Summary of the results. Germ J Psychol 9: 319-339

Dowling GP, McDonough ET, Bost RO (1986): "Eve" and "Ecstasy": A report of five deaths associated with the use of MDEA and MDMA. JAMA 257:1615-1617

Fahrenberg J, Selg H, Hampel R (1984): Das Freiburger Persönlichkeitsinventar FPI-R. Göttingen, Hogrefe

Gibb JW, Stone D, Johnson M, Hanson GR (1990): Neurochemical effects of MDMA. In Peroutka SJ (ed), Ecstasy: The Clinical, Pharmacological and Neurotoxicological Effects of the Drug MDMA. Boston, Kluwer, pp 133-150

Gouzoulis E, Bardeleben Uv, Rupp A, Kovar KA, Hermle L (1992a): Neuroendocrine and cardiovascular effects of 3,4-methylenedioxyethamphetamine (MDE, "Eve") in healthy volunteers. (In press)

Gouzoulis E, Steiger A, Ensslin M, Kovar KA, Hermle L (1992b): Sleep EEG effects of 3,4-methylenedioxyethamphetamine (MDE, "Eve") in healthy volunteers. Biol Psychiatry (In press)

Gouzoulis E, Borchardt D, Hermle L (1992c): A case of toxic psychosis induced by "Eve" (3,4-methylenedioxyethamphetamine). Arch Gen Psychiatry (In press)

Greer G, Tolbert R (1986): Subjective reports of the effects of MDMA in a clinical setting. J Psychoactive Drugs 18:319-327

Hermle L, Fünfgeld M, Oepen G, Botsch H, Borchardt D, Gouzoulis E, Spitzer M (1992): Mescaline-induced psychopathological, neuropsychological and neurometabolic effects in normal subjects. Biol Psychiatry (In press)

Johnson M, Letter AA, Merchant K, Hanson GR, Gibb JW (1988): Effects of 3,4-methylenedioxyamphetamine and 3,4-methylenedioxymethamphetamine isomers on central serotonergic, dopaminergic and nigral neurotensin systems of the rat. J Pharmacol Exp Ther 244:977-982

Laux L, Glanzmann P, Schaffner P, Spielberger CD (1981): Das State-Trait-Angstinventar (STAI). Weinheim, Beltz

Nichols DE (1986): Differences between the mechanism of action of MDMA, MBDB, and the classic hallucinogens. Identification of a new therapeutic class: Entactogens. J Psychoactive Drugs 18:305-313

Peroutka SJ (1990): Recreational use of MDMA. In Peroutka 
SJ (ed.), Ecstasy: The Clinical, Pharmacological and Neurotoxicological Effects of the Drug MDMA. Boston, Kluwer, pp 53-62

Ricaurte G, De Lanney LE, Irwin I, Langston JW (1988): Toxic effects of MDMA on central serotonergic neurons in the primate: importance of route and frequency of drug administration. Brain Res 446:165-168

Schmidt CJ (1987): Acute administration of methylenedioxymethamphetamine: comparison with the neurochemical effects of its N-desmethyl and N-ethyl analogs. Eur J Pharmacol 136:81-88

Seymour RB (1986): MDMA. San Francisco, Haight Ashbury Publications

Shulgin AT (1986): The background and chemistry of MDMA. J Psychoactive Drugs 18:291-304
Stone DM, Johnson M, Hanson GR, Gibb GW (1987): Acomparison of the neurotoxic potential of methylenedioxyamphetamine (MDA) and its N-methylated and N-ethylated derivatives. Eur J Pharmacol 134:245-248

von Zerssen D (1986): Clinical Self-Ratings-Scales (CSRS) of the Munich Psychiatric System(PSYCHISMünchen). In Sartorius N, Bau TA (eds), Assessment of Depression. Berlin

Whitaker-Azmitia PA, Aronson T (1989): Panic attacks associated with MDMA (Ecstasy). Am J Psychiatry 146:119

Wittchen HU, Schramm E, Zaudig M, Spengler P, Rummler B, Mombour W, Hiller W (1987): SKID, Strukturiertes Klinisches Interview for DSM-III-R. Weinheim, Beltz 